\title{
Output Voltage and Efficiency of Novelty Architecture of Charge Pump versus Clock Frequency and MOSFETs Sizes
}

\author{
David Matoušek \\ Department of Electrical Engineering \\ University of Pardubice \\ 53002 Pardubice, Czech Republic \\ david.matousek@upce.cz
}

\begin{abstract}
Charge pump is circuit that produces voltage higher than supply voltage or negative voltage. Today, charge pumps became an essential parts of electronic equipment. The integration of charge pumps directly into the target system allows manufacturers to feed a complex system with many specific power requirements from a single source. However, charge pump efficiency is relatively small. This paper is devoted to questions of efficiency of presented variant of charge pump. Thus efficiency as dependence on number of stages, clock frequency, output current and MOSFETs sizes of presented charge pump was simulated. The aim of this study is determination of MOSFETs sizes and theirs influence to efficiency and output voltage. Complex optimization of this circuit will follow in the next period.
\end{abstract}

Keywords-2-phase Charge Pump; efficiency; switch size determination.

\section{INTRODUCTION}

Generally known variant of charge pump is Dickson Charge Pump (DCP) [1]. Design rules for DCP are summarized in [2]. The differential voltage $\Delta V$ between nodes $n$ and $n+1$ is

$$
\Delta V=V_{n+1}-V_{n}=V_{E}-V_{T}
$$

where $V_{E}$ is voltage gain, $V_{T}$ is threshold voltage.

Value of voltage gain decreased by stray capacitance of node as follows

$$
V_{E}=\left(\frac{C_{T}}{C_{T}+C_{S}}\right) \cdot V_{C L K}
$$

where $V_{E}$ is voltage gain, $C_{T}$ is transfer capacitance, $C_{S}$ is stray capacitance, $V_{C L K}$ is amplitude of clocks.

Since the output voltage applies according

$$
V_{\text {OUT }}=V_{I N}+N \cdot\left(V_{E}-V_{T}\right)-V_{T}
$$

where $V_{\text {OUT }}$ is output voltage, $V_{I N}$ is input voltage, $N$ is number of stages, $V_{E}$ is voltage gain, $V_{T}$ is threshold voltage.

The published research results were supported by the Internal Grant Agency of University of Pardubice, the project SGFEI 2016.

\author{
Jiří Hospodka ${ }^{1}$, Ondřej Šubrt ${ }^{1,2}$ \\ ${ }^{1}$ Department of Circuit Theory \\ FEE CTU in Prague, Czech Republic \\ ${ }^{2}$ ASICentrum, a company of the Swatch Group \\ Prague, Czech Republic
}

The threshold voltage of used transistors has usually cardinal effect to resulted value of the output voltage. This parameter limits the DCP implementation, especially for supply voltage lower than $1 \mathrm{~V}$. Therefore, sub-volt applications must use other circuit solutions of charge pumps [3], [4].

Generally used principle for the threshold effect suppression is a change of connection of the transfer transistor from diode mode to switch mode [5], [6], [7]. For this solution, voltage drop between two nodes is decreased from the threshold gate-source voltage to the saturation voltage of channel, only.

\section{PRESENTED VARIANT OF Charge PumP}

Presented variant of charge pump [8] uses 2-phase clocks. One cell of this charge pump is shown in Fig. 1. The cell consists of five transistors $\left(M_{1}\right.$ to $\left.M_{5}\right)$ and transfer capacitor $\left(\mathrm{C}_{\mathrm{T}}\right)$.

This block is driven by clock signals according to Fig. 2.

In the first phase $\left(C L K 1=V_{D D}, C L K 2=V_{D D}\right)$, transistors $M_{1}, M_{3}$ and $M_{5}$ are closed. Thus transfer capacitor $\mathrm{C}_{\mathrm{T}}$ is biased to supply voltage $\mathrm{V}_{\mathrm{DD}}$.

In the second phase (CLK1 $=$ GND, CLK2 $=$ GND), transistors $\mathrm{M}_{2}$ and $\mathrm{M}_{4}$ are closed. Thus transistor $\mathrm{M}_{2}$ holds bias transistor $\mathrm{M}_{5}$ in disconnected state. Transistor $\mathrm{M}_{4}$ connects the transfer capacitor $\mathrm{C}_{\mathrm{T}}$ between input and output of cell. Therefore, input voltage is increased by a voltage of transfer capacitor from previous (biased) phase.

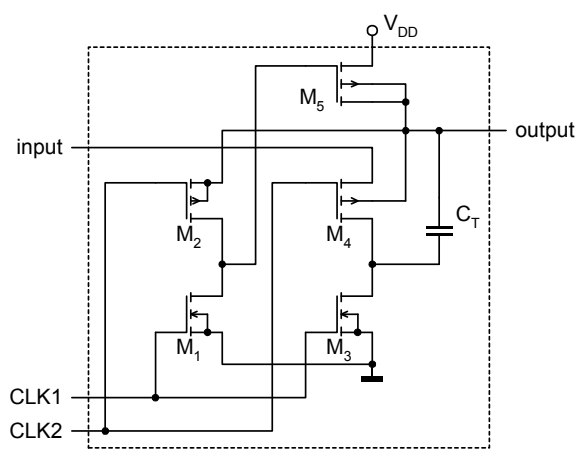

Figure 1. One cell of proposed charge pump [8]. 
In the last phase $\left(\mathrm{CLK} 1=\mathrm{GND}, \mathrm{CLK} 2=\mathrm{V}_{\mathrm{DD}}\right)$, all transistors are opened.

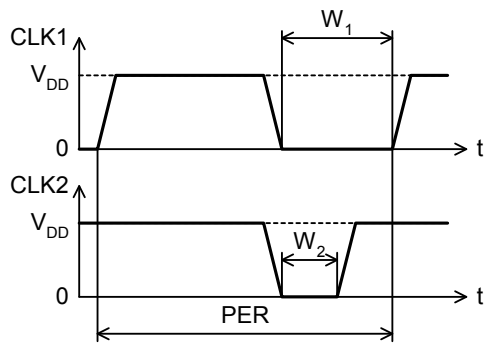

Figure 2. Waveforms of 2-phase clocks.

Driving clocks from Fig. 2 have overlapped character. Symbols $W_{1}$ and $W_{2}$ mark width of pulses both clock signals. $P E R$ is period. The optimal values of $W_{1}, W_{2}$ were estimated in previous period [8].

\section{DESIGN ALGORITHM}

The design rules for estimation of parameters of presented charge pump is summarized to undermentioned steps. We consider these charge pump specifications:

- Power supply voltage $\mathrm{V}_{\mathrm{DD}}=0.7 \mathrm{~V}$, minimal steady output voltage $\mathrm{V}_{\text {OUT }}=4 \mathrm{~V}$,

- Output load capacitance $\mathrm{C}_{\mathrm{L}}=300 \mathrm{pF}$, output current $\mathrm{I}_{\mathrm{L}}=4 \mu \mathrm{A}$,

- Maximal output voltage ramp-up time $t_{r}=150 \mu \mathrm{s}$.

A. The number of stages

Number of stages $N$ was calculated as ratio of output voltage $V_{O U T}$ and voltage gain of one stage $V_{E}$. Result value had to be an integer:

$$
N=\frac{V_{\text {OUT }}}{V_{E}}=\frac{4}{0.7}=5.714=6
$$

\section{B. The clock frequency}

Initial clock frequency was selected as $f_{C L K}=20 \mathrm{MHz}$.

\section{The size of transfer capacitor}

Value of transfer capacitance $C_{T}$ we calculated from known load capacitance $C_{L}$, number of stages $N$, ramp-up time $t_{r}$ and clock frequency $f_{C L K}$ [2]:

$$
\begin{aligned}
& C_{T}=C_{L} \frac{N}{t_{r} \cdot f_{C L K}}= \\
& =300 \cdot 10^{-12} \frac{6}{150 \cdot 10^{-6} \cdot 20 \cdot 10^{6}}=0.6 \mathrm{pF}
\end{aligned}
$$

\section{The W/L of used transistors}

The MOSFETs $M_{3}$ to $M_{5}$ and $M_{D}$ are used for transfer charge. Thus these transistors should have conductance at least ten times higher than conductance matched to the output current [2]. Initial size of these MOSFETs must be estimated from I-V characteristics, only.
The MOSFETs $M_{1}$ to $M_{2}$ cause losses of charge, but these MOSFETs are used for driving transistor $\mathrm{M}_{5}$ only. Therefore, these MOSFETs must be designed relatively narrow.

Parameters of used transistors are summarized in Table I.

TABLE I. PARAMETERS OF TRANSISTORS

\begin{tabular}{|c|c|c|c|}
\hline Transistor & $\mathbf{W}(\boldsymbol{\mu m})$ & $\mathbf{L}(\boldsymbol{\mu m})$ & model \\
\hline $\mathrm{M}_{1}$ & 0.2 & 0.1 & nmos_hvt \\
\hline $\mathrm{M}_{2}$ & 1 & 0.1 & pmos_hvt \\
\hline $\mathrm{M}_{3}$ & 0.5 & 0.1 & nmos_hvt \\
\hline $\mathrm{M}_{4}$ & 2.5 & 0.1 & pmos_hvt \\
\hline $\mathrm{M}_{5}$ & 2.5 & 0.1 & pmos_hvt \\
\hline $\mathrm{M}_{\mathrm{CT}}$ & 30 & 10 & nmos_hvt \\
\hline $\mathrm{M}_{\mathrm{D}}$ & 20 & 0.8 & nmos_na18v \\
\hline $\mathrm{M}_{\text {BUFA }}$ & 5 & 0.1 & nmos_hvt \\
\hline $\mathrm{M}_{\text {BUFB }}$ & 12.5 & 0.1 & pmos_hvt \\
\hline
\end{tabular}

Transfer capacitor $\mathrm{C}_{\mathrm{T}}$ is realized as transistor $\mathrm{M}_{\mathrm{CT}}$ (we do not use MIM capacitors), thus capacity of this MOSFET must be calculated from derivation of I-V characteristics.

$\mathrm{M}_{\mathrm{BUFA}}$ and $\mathrm{M}_{\mathrm{BUFB}}$ are used as clock buffers. Models nmos_hvt and pmos_hvt correspond to "high voltage" transistors with relatively high value of $\mathrm{V}_{\mathrm{T}}$. Model nmos na18v corresponds to native transistor for $1.8 \mathrm{~V}$ technology.

Estimated area of chip for realization of proposed charge pump is listed in Table II, where $N$ is number of stages, $A_{S T A G E S}$ is area for realization of stages, $A_{B U F D E T}$ is area for realization clock buffers and output detector, $A_{\text {TOTAL }}$ is area for realization proposed charge pump with required number of stages.

TABLE II. ESTIMATED AREA FOR REALIZATION OF CHARGE PUMP

\begin{tabular}{|c|c|c|c|}
\hline $\mathbf{N}$ & Astages $\left(\boldsymbol{\mu \mathbf { m } ^ { 2 } )}\right.$ & $\mathbf{A}_{\text {Bufdet }}\left(\boldsymbol{\mu \mathbf { m } ^ { \mathbf { 2 } } )}\right.$ & $\mathbf{A}_{\text {Total }}\left(\boldsymbol{\mu \mathbf { m } ^ { 2 }}\right)$ \\
\hline 1 & 469 & 30 & 499 \\
\hline 2 & 998 & 30 & 1028 \\
\hline 3 & 1497 & 30 & 1527 \\
\hline 4 & 1996 & 30 & 2026 \\
\hline 5 & 2495 & 30 & 2525 \\
\hline 6 & 2994 & 30 & 3024 \\
\hline
\end{tabular}

\section{Study OF Clock FreQUenCy INFLUENCE TO} EFFICIENCY AND OUTPUT VOLTAGE

The aim of this part of simulations is to determine the influence of clock frequency to efficiency and output voltage.

Block diagram of simulated charge pump is shown in Fig. 3. It is 6-stage charge pump $(\mathrm{N}=6)$. For other cases, number of stages are changed, only. Both clock signals are buffered by invertors with strong output stage. The last stage is a diode detector based on transistor $M_{D}$. Output load is modeled by resistor $R_{L}$ and capacitor $\mathrm{C}_{\mathrm{L}}$. Presented charge pump was powered from $V_{D D}=0.7 \mathrm{~V}$ and both clock signals had the same amplitude $0.7 \mathrm{~V}$, too. Symbol $I_{S}$ marks consumed current. Output voltage at load is marked $V_{\text {OUT }}$. 




Figure 3. Simplified schematic diagram of simulated charge pump for $\mathrm{N}=6$.

Number of stages $\mathrm{N}$ was varied from 1 to 6 , clock frequency was set to values 10, 13.3, 20, 27, $40 \mathrm{MHz}$.

Efficiency and output voltage dependency to the clock frequency and output current $\mathrm{I}_{\mathrm{L}}$ are shown in Fig. 4 and Fig. 5.

Total efficiency calculates by (6)

$$
\zeta=\frac{V_{O U T}^{2}}{V_{D D} \cdot I_{S} \cdot R_{L}} \cdot 100 \%,
$$

where $V_{O U T}$ is output voltage, $V_{D D}$ is power voltage, $I_{S}$ is consumed current, $R_{L}$ is output load.

Efficiency was varied from $38.47 \%$ at frequency $10 \mathrm{MHz}$ to $28.64 \%$ at frequency $40 \mathrm{MHz}$ for nominal value of output current $I_{L}=4 \mu \mathrm{A}$. For the same conditions, output voltage varies from $3.741 \mathrm{~V}$ to $4.051 \mathrm{~V}$

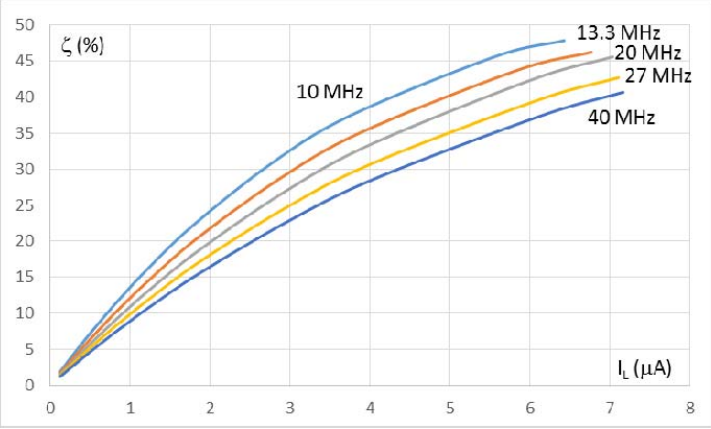

Figure 4. Efficiency as function of $I_{L}$ for $\mathrm{N}=6$

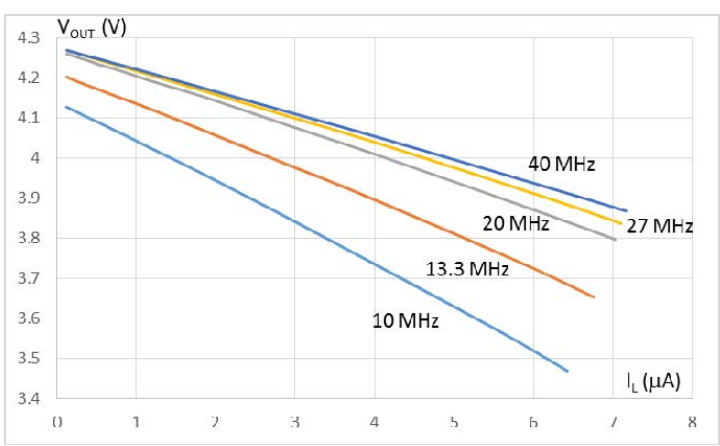

Figure 5. Output voltage as function of $I_{L}$ for $\mathrm{N}=6$.
Simulation results for other number of stages $(\mathrm{N}$ varies from 1 to 6 ) of charge pump are summarized in Table III.

TABLE III. EFFICIENCY AND OUTPUT VOLTAGE FOR VARIOUS NuMBER OF STAGES AT OUTPUT CURRENT $4 \mu \mathrm{A}$

\begin{tabular}{|c|c|c|}
\hline $\mathbf{N}$ & $\boldsymbol{\zeta ( \% )}$ & Vout $(\mathbf{V})$ \\
\hline 1 & 69.53 to 59.33 & 1.275 to 1.290 \\
\hline 2 & 64.63 to 43.26 & 1.919 to 1.950 \\
\hline 3 & 60.06 to 47.07 & 2.540 to 2.590 \\
\hline 4 & 53.75 to 42.02 & 3.130 to 3.120 \\
\hline 5 & 47.79 to 37.30 & 3.638 to 3.762 \\
\hline 6 & 37.47 to 28.64 & 3.671 to 4.051 \\
\hline
\end{tabular}

\section{MOSFETS SIZE INFLUENCE TO EFFICIENCY} AND OUTPUT VOLTAGE

The aim of this part of simulations is to determine influence of MOSFETs size to efficiency and output voltage. Thus sizes of all transistors were swept, but only one parameter was changed in one time, other sizes according Table I were left unchanged. Output load was set to constant value $R_{L}=1 \mathrm{M} \Omega$. These simulations were performed for 6-stage charge pump only.

Next, the six variants of simulations are performed to determine influence of transistor dimensions.

Variant \#1: widths of transistors $\mathrm{M}_{4}$ and $\mathrm{M}_{5}$ were swept from $0.5 \mu \mathrm{m}$ to $50 \mu \mathrm{m}$. The sizes of these transistors has the key influence to output voltage and efficiency, see Fig. 6.

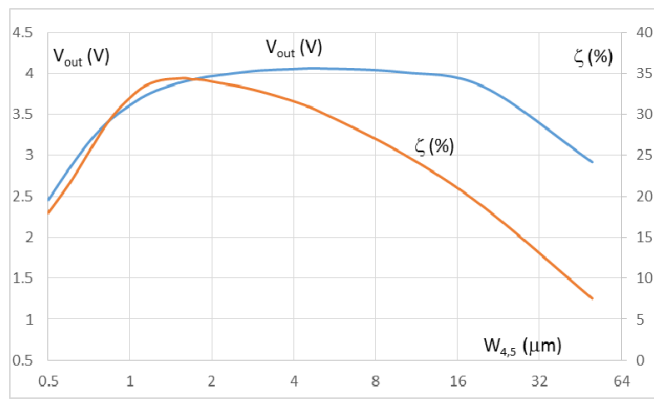

Figure 6. Variant 1: Efficiency and output voltage as functions of $W_{4,5}$.

Variant $\# 2$ : width of detector $\mathrm{M}_{\mathrm{D}}$ was swept from $0.5 \mu \mathrm{m}$ to $50 \mu \mathrm{m}$. The size of this transistor has primarily influence to the efficiency, see Fig. 7.

Variant \#3: width of transistor $\mathrm{M}_{3}$ was swept from $0.2 \mu \mathrm{m}$ to $10 \mu \mathrm{m}$. The size of this transistor has primarily influence to the output voltage, see Fig. 8. 
Variant \#4 and \#5: widths of transistors $\mathrm{M}_{1}$ and $\mathrm{M}_{2}$ were swept from $0.2 \mu \mathrm{m}$ to $10 \mu \mathrm{m}$ or from $0.2 \mu \mathrm{m}$ to $20 \mu \mathrm{m}$ respectively. It is clear evident that transistors $\mathrm{M}_{1}$ and $\mathrm{M}_{2}$ must be narrowed, see Fig. 9.

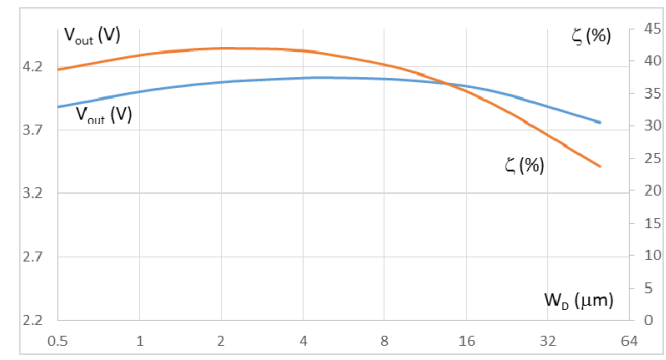

Figure 7. Variant 2: Efficiency and output voltage as functions of $W_{D}$.

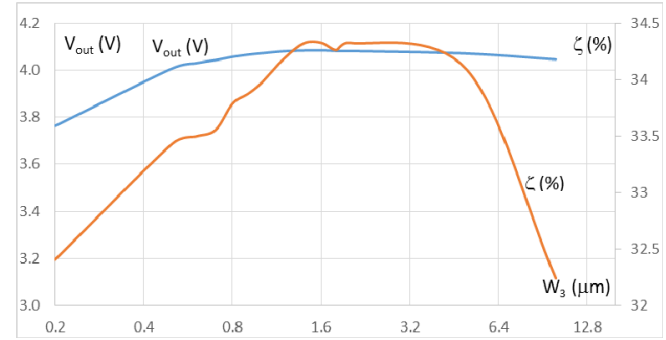

Figure 8. Variant 3: Efficiency and output voltage as functions of $W_{3}$.

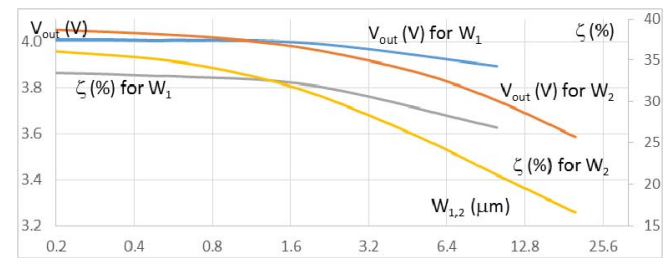

Figure 9. Variant 4, 5: Efficiency and output voltage as functions of $W_{1,2}$.

Variant \#6: width of transistor $\mathrm{M}_{\mathrm{CT}}$ was swept from $6 \mu \mathrm{m}$ to $60 \mu \mathrm{m}$. The optimal size of this transistor is a compromise between efficiency and output voltage, see Fig. 10.

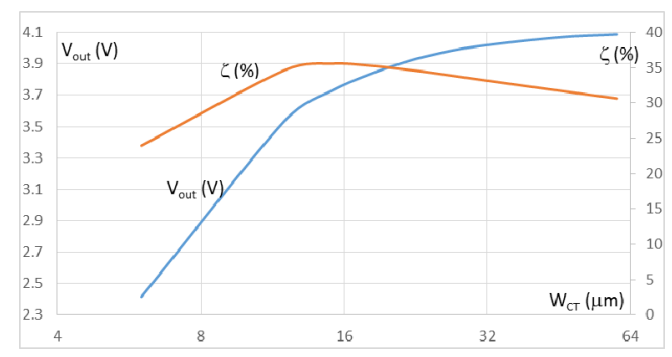

Figure 10. Variant 6: Efficiency and output voltage as functions of $W_{C T}$.

Variant \#7: width of transistor $\mathrm{M}_{\mathrm{BUFA}}$ was swept from $1 \mu \mathrm{m}$ to $20 \mu \mathrm{m}$ and simultaneously width of transistor $\mathrm{M}_{\mathrm{BUFB}}$ was swept from $5 \mu \mathrm{m}$ to $100 \mu \mathrm{m}$. Maximal value of efficiency $\zeta=33.67 \%$ for $V_{\text {OUT }}=4.006 \mathrm{~V}$ occurs for $W_{B U F A}=1 \mu \mathrm{m}$ and $W_{B U F B}=5 \mu \mathrm{m}$. This variant isn't plotted, because differences of values of efficiency and output voltage were relatively small.

\section{CONCLUSION}

Study of efficiency and output voltage versus clock frequency and MOSFETs sizing for proposed charge pump was performed. Results from this study can be used for complex optimization study performed in the next period.

Parameters of MOSFETs and clock scheme were chosen according to previous study [8]. Analysis were performed by Eldo simulator version 2010.2b from Mentor Graphics Corporation.

From the first part of simulations we can see that the efficiency increases with increasing load current and decreasing clock frequency. And reversely, the output voltage increases with decreasing load current and increasing clock frequency.

Results from the second part of simulations is the key for optimization efficiency of presented charge pump. Values of efficiency and output voltage are $\zeta=33.43 \%$ and $V_{\text {OUT }}=4.008 \mathrm{~V}$ for original sizes of MOSFETs according to Table I and load $R_{L}=1 \mathrm{M} \Omega$.

The transistor sizing technique allows to improve the efficiency about ten percent. Dimensions of transistors $\mathrm{M}_{4}, \mathrm{M}_{5}, \mathrm{M}_{\mathrm{D}}$ and $\mathrm{M}_{\mathrm{CT}}$ have the key effect to efficiency, $\mathrm{M}_{3}$ has important effect to the output voltage. Effect of other transistors has predictable character and we may neglect effect of size of these transistors.

The presented results are limited to pre-layout simulations only. The parasitic effects of higher orders (e.g. interlayer capacitance, metallic interconnection capacitance etc.) are neglected.

\section{REFERENCES}

[1] J. F. Dickson, "On-Chip high-voltage generation in NMOS integrated circuits using an improved voltage multiplier technique", IEEE Journal of Solid-State Circuits, vol. 11, no. 3, pp. 374-378, 1976.

[2] F. Pan, T. Samaddar, "Charge pump circuit design", McGraw-Hill, New York, 2006.

[3] G. Palumbo, D. Pappalardo, "Charge pump circuits: An overview on design strategies and topologies", IEEE Circuits and Systems Magazine, First Quarter 2010, 1531-636X/10, IEEE 2010, pp. 31-45.

[4] D. Matousek, "Comparison of selected architectures of negative Charge Pumps with new design", In: Proceedings of the 24th International Conference Radioelektronika, Bratislava, 2014, pp. 1-4.

[5] T. Yamazoe, H. Ishida, Y. Nihongi, "A Charge Pump that generates positive and negative high voltages with low power-supply voltage and low power consumption for nonvolatile memories", In: International Symposium ISCAS, Taipei, Taiwan, 2009, pp. 988-991.

[6] O.-Y. Wong, R. Wong, W.-S. Tam, C.-W. Kok, “An overview of charge pumping circuits for Flash memory applications", In: Proceeding of the 9th International Conference ASICON, Xiamen, China, pp. 116-119, 2011.

[7] O.-Y. Wong, H. Wong, W.-S. Tam, C.-W. Kok, "On the design of power- and area-efficient Dickson charge pump circuits", Analog Integr Circ Sig Process, 2014, pp. 373-389.

[8] D. Matousek, O. Subrt, J. Hospodka, "Charge Pump Design for Use in NVM Device Test and Measurement", In: MEASUREMENT 2015, Proceedings of the 10th International Conference, Smolenice, 2015, pp. 203-206. 